\title{
ROOTS AND BRANCHES OF THE LINGNAN SCHOOL
}

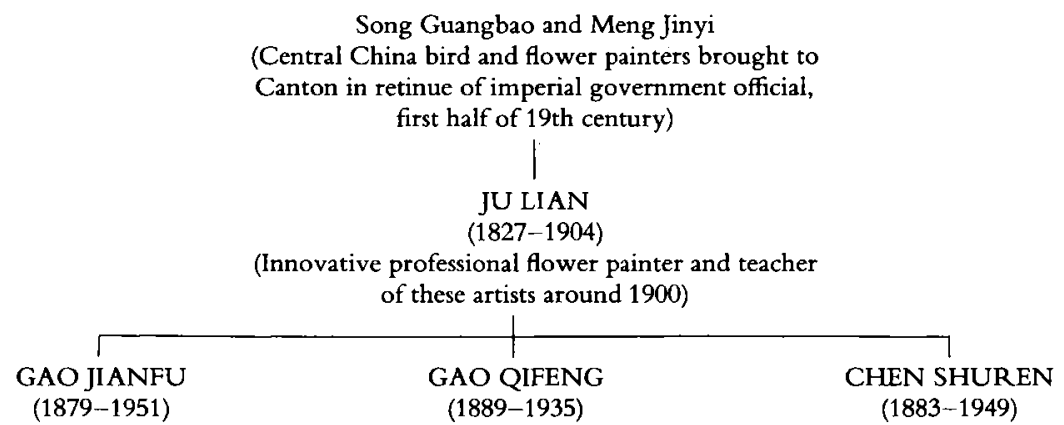

The three founders of the Lingnan School study in Japan, 1906-1911, and participate in<smiles>C1=CC=C1</smiles>

Disciples at Spring

Slumber Studio in

Canton, 1924-1937
Republican Revolution of 1911

Disciples at Heavenly

Breeze Pavilion in

Canton, 1929-1935

\begin{tabular}{ccccc|} 
Remain in China & To Hong Kong & To Taiwan & Overseas & Zhang Kunyi \\
Guan Shanyue & Li Fuhong & You Yunshan & Situ Qi & Huang Shaoqiang \\
Li Xiongcai & Yang Shanshen & (Ven. Xiao Yun) & & Zhou Yifeng \\
Su Wonong & & & & He Qiyuan \\
Fang Rending & & & & Zhao Shaoang
\end{tabular}

No direct followers

Numerous disciples in Hong Kong since 1950s (Today Art Association)

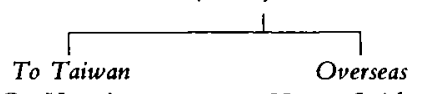

Ou Haonian

Huang Leisheng

Liu Yunheng (Stephen Lowe) She Miaozhi 
